\title{
Improvements to Secondary Windings of Tesla Transformers
}

\author{
Richard M Craven ${ }^{1}$, Ivor R Smith ${ }^{1} \&$ Bucur M Novac $^{1}$ \\ ${ }^{1}$ Wolfson School of Mechanical, Electrical and Manufacturing Engineering, Loughborough University, \\ Leicestershire LE11 3TU, UK \\ Correspondence: Ivor R Smith, Wolfson School of Mechanical, Electrical and Manufacturing Engineering, \\ Loughborough University, Leicestershire LE11 3TU, UK. E-mail: I.R.Smith@lboro.ac.uk
}

Received: November 21, 2016

Accepted: January 12, 2017

Online Published: January 23, 2017

doi:10.5539/apr.v9n1p93

URL: http://dx.doi.org/10.5539/apr.v9n1p93

\begin{abstract}
Impulse excitation of the tuned primary circuit of a Tesla transformer generates a voltage and current response in the similarly tuned secondary circuit that contains both a fundamental component and a series of multiple higher-order modes. This paper investigates the most significant of these modes, in order to demonstrate a design approach that, when applied to the secondary winding, can bring about a reduction in the higher-order modes without significantly affecting the fundamental term. The resulting process leads to an improved spectral purity of the transformer output, making it better suited than existing conventional designs for application in electronic warfare and other high-power systems.
\end{abstract}

Keywords: Tesla transformers, high-voltage techniques, pulsed power supplies, harmonic reduction.

\section{Introduction}

A wide range of experimental topics in both physics and engineering requires extremely fast rising pulses with amplitudes greater than $100 \mathrm{kV}$. This is frequently provided via the use of a Tesla transformer (Glascoe \& Lebacqz, 1948; Serjeant \& Dollinger, 1989), which transfers energy from a low impedance primary circuit to a higher impedance secondary circuit to bring the voltage of the secondary circuit to the much higher value required by a typical load. As evident from Figure 1, the two circuits are capacitively tuned to the same resonant frequency when in isolation. The lower end of the secondary winding is normally earthed and a sharpening gap included in series with the high-voltage output line when the load voltage is required to be fast rising. The transformer is energised by closure of the primary switch $\mathrm{S}$, often in the form of a spark gap.

Although the underlying concepts were clearly presented over a hundred years ago, the Tesla transformer still plays a vital role in meeting important pulse generation requirements in technically active countries worldwide. Typically important new applications for Tesla transformers are outlined elsewhere (Efremov, Koshelev, Kovalchuk, Plisko \& Sukhushin, 2011; Zhao, et al, 2013;Wang, Novac, Pecastaing \& Smith, 2016).

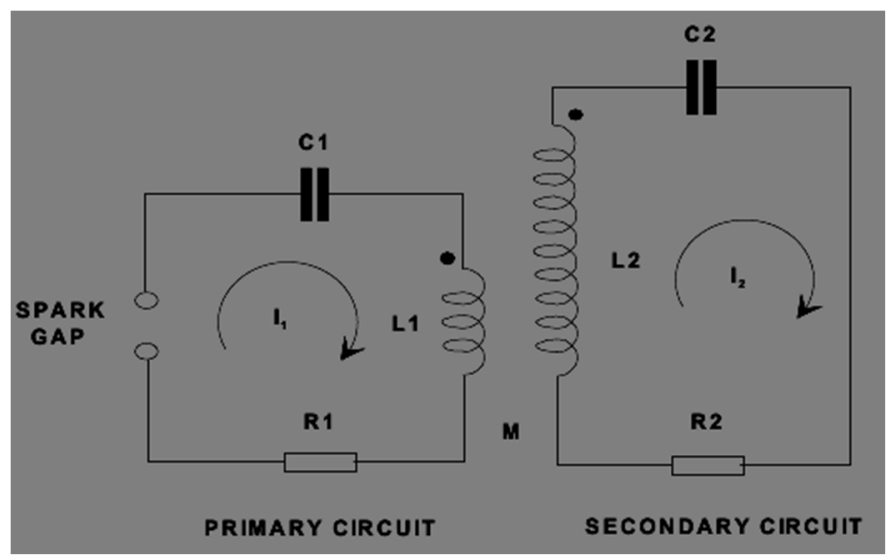

Figure 1: Equivalent circuit of a Tesla transformer (field grading electrode and load not shown) 
Although totally unexpected, the secondary windings of Tesla transformers have considerable similarities with those of coaxial helical filters, as used in radio frequency applications to filter the output from radio receivers and cellular phone base stations. This considerable similarity stimulated the possibility of developing a novel secondary winding design for Tesla transformers during an ongoing research programme at Loughborough University (Craven, 2014). The aim was to reduce the higher-order modes normally present in the output and so provide important advantages for both high-power military and civilian applications.

\section{Helical Filters}

Helical filters (Zverev, 1967) are normally constructed in the form of a cylindrical highly-conductive air filled cavity made from copper and often silver plated. A helically-wound single-layer solenoid, mounted along the central axis, is earthed at one end and resonates with a capacitor series-connected to the other end of the structure. Coupling between the input and output signals and the helical coil is achieved via an arrangement of loops. The effective electrical length of the helix is a little below the quarter-wave length that corresponds to its resonant frequency, with less electric field energy stored in the distributed capacitance of the helix than in the associated magnetic field. The additional electric energy required to bring the overall structure into resonance is provided by the top-loading capacitor. The resonant structure formed along the central axis of the cavity is earthed at one end and at frequencies not above VHF is often wound as a self-supporting air-cored single-layer solenoid. An arrangement of several helical filters tuned to slightly different frequencies can be assembled to form a filter with very accurately controlled passband and stopband frequencies.

\section{Background Considerations}

During an investigation into ways by which standing waves could be suppressed on a $\lambda / 4$ resonant structure, Vitzmuller (1987) established that at resonance the electric field set up by the solenoidal coil of a resonant helical filter varies sinusoidally between a minimum at its base and a maximum at its upper end. The magnetic field of the solenoid varies conversely. The effect of a reversal in the winding direction of a portion of the helical turns is a reduction in the effective electrical length to less than a quarter of the wave-length $\lambda$ that corresponds to the original resonant frequency. As in the Tesla secondary circuit, resonance is restored by a change in the capacitance series-connected to the upper end of the solenoid, so that the total electrical field energy is again equal to the total stored magnetic field energy. In general, at a fundamental resonant frequency $f_{1}$ the spatial difference between the conditions at the two ends of the winding must be a quarter of the resonant wavelength $(\lambda / 4)$ and all of a series of odd multiples thereof. This gives rise to a standing wave along the coil, which contains a series of related wave-lengths for which the equivalent electrical lengths of the coil are:

$$
\lambda / 4 \quad 3 \lambda / 4 \quad 5 \lambda / 4 \ldots \ldots(2 \mathrm{n}+1) \lambda / 4 \quad \text { where } \mathrm{n}=1,2, \ldots
$$

Figure 2 shows the normalized voltage distribution along the length $\mathrm{H}$ of the coil for the fundamental and the first three higher-harmonic odd modes. In general, for all odd numbered modes $\mathrm{n}$, minimum voltage nodes are present at the base of the coil $(\mathrm{H}=0)$ and locations not greater than $\mathrm{H}$ and specified by

$$
\begin{array}{llll}
0 & 2 \mathrm{H} / \mathrm{n} & 4 \mathrm{H} / \mathrm{n} & 6 \mathrm{H} / \mathrm{n} . \ldots . . \mathrm{H}
\end{array}
$$

while maximum voltage anti-nodes occur between the base and the top of the coil at locations specified by

$$
\mathrm{H} / \mathrm{n} \quad 3 \mathrm{H} / \mathrm{n} \quad 5 \mathrm{H} / \mathrm{n} . \ldots . . \mathrm{H}
$$

The series of standing wave terms identified above correspond to the frequencies $f_{1}, f_{3}, f_{5}$ etc. of the sinusoidal odd harmonic currents that can flow in the coil.

During his investigation into ways by which standing waves could be suppressed on a $\lambda / 4$ resonant structure, (Vitzmuller, 1987) investigated a helical coil of length $H$ in which a length $H_{1}$ was wound in the forward direction and a length $\mathrm{H}_{2}$ in the reverse direction. He established theoretically that the fundamental resonant frequency of the overall structure remained corresponding to $\lambda / 4=\mathrm{H}_{1}+\mathrm{H}_{2}$, rather than to an increased frequency corresponding to $\lambda / 4$ alone, a feature confirmed experimentally by the present authors. However, the frequencies and amplitudes of the higher-order modes are sensitive to the ratio $\mathrm{H}_{2} / \mathrm{H}_{1}$, providing a basis on which they can be controlled, whilst leaving those of the fundamental frequency substantially unchanged. It was this feature that was utilized to improve the spectral purity control of the output from a Tesla transformer. 
$f_{1}$

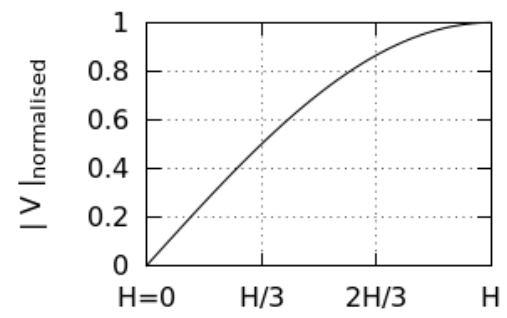

$f_{5}$

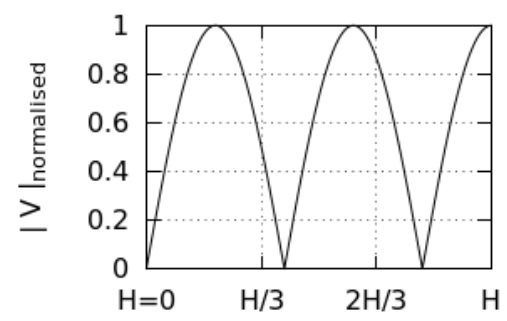

$f_{3}$

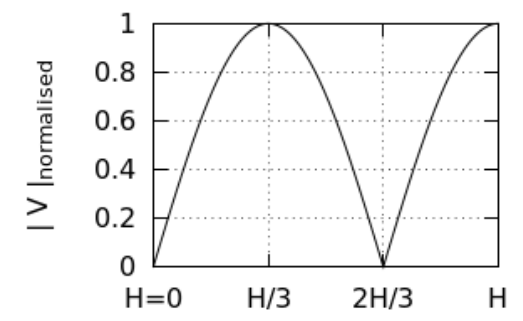

$f_{7}$

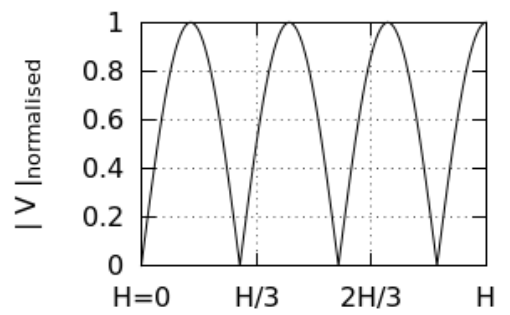

Figure 2. Sinusoidal voltage distributions of the fundamental and first three higher-order modes present

\section{Methodology}

During the investigation at Loughborough, a range of helical coils were manufactured to enable the effect of the turn's reversal to be studied in practice. The coils were wound on acrylonitrile butadiene styrene (ABS) tubes of $1 \mathrm{~mm}$ wall thickness, $570 \mathrm{~mm}$ long and $114 \mathrm{~mm}$ outer diameter. All the 1600 enamelled copper wire turns of one coil (termed the $0 \%$ coil) were wound in the same direction, while the other four coils were identical in overall size but with $10 \%, 22.5 \%, 33 \%$ and $50 \%$ of the number of turns from one end reversed in direction. Together with an accompanying computer program, these coils helped to identify the optimum number of reversed turns that maximised the effect produced on the higher-order modes in comparison with the substantially unchanged fundamental mode.

\subsection{Quality Factor Measurements}

The fundamental resonant frequency $\mathrm{f}_{1}$ and those for the first three higher odd mode frequencies $\mathrm{f}_{3}, \mathrm{f}_{5}$ and $\mathrm{f}_{7}$ were calculated from the estimated self-inductance and self-capacitance of the experimental secondary winding coils and confirmed by measurement. Each of the experimental coils was then energized in turn from a constant current source swept over a frequency range that embraced the first four modes. The quality factor $\left(\mathrm{Q}_{\mathrm{m}}\right)$ of the coils at the resonant frequency of each mode $\left(f_{m}\right)$ is the ratio of the energy lost to the energy stored per cycle at that frequency, when the complex input impedance is wholly real. From the specific single frequencies above and below the resonant frequency ( $f_{\mathrm{mu}}$ and $\mathrm{f}_{\mathrm{ml}}$ respectively, the half power points), at which the magnitudes of the real and imaginary components of the impedance are equal and the $\mathrm{Q}$ factor is (Terman,1955):

$$
\mathrm{Q}_{\mathrm{m}}=\mathrm{f}_{\mathrm{ml}} /\left(\mathrm{f}_{\mathrm{mu}}-\mathrm{f}_{\mathrm{ml}}\right)
$$

Determining the $\mathrm{Q}$ factor for a mode frequency enables the effect to be established that any reduction in it will have on the corresponding current circulating in the equivalent circuit of the coil (Craven, 2014).

\subsection{In-Circuit Spectrum Measurements}

Table 1 lists the parameters of an experimental Tesla transformer designed to produce a reliable reference, using which the responses of a conventionally-wound secondary coil and coils with a proportion of the turns reversed could be tested and compared with one another. Each secondary coil was top loaded by a field-grading electrode, with the resonant frequencies of the primary and secondary circuits retuned to maintain their resonant conditions. A low current $10 \mathrm{kV}$ dc to de converter power supply allowed reliable and repeatable firing to occur at low repetition rates, enabling the different 'single shot' secondary coil mode frequency current measurements to be characterised.

All experimental measurements of the transformer response were obtained at a fixed location within a controlled laboratory environment. Numerous time-domain waveforms of the secondary current were captured, using a wideband current transformer and a digital storage oscilloscope providing $100 \mathrm{MHz}$ of instrumentation bandwidth. Initial measurements were made using the reference coil, after which the other coils were substituted 
in turn and the response measurements repeated. The information was numerically processed via a Fast Fourier Transform (FFT) to provide a frequency domain spectrum. The actual mode resonant frequencies and amplitudes were observed for the $\mathrm{f}_{1}, \mathrm{f}_{3}$, etc. modes of the $0 \%$ reference coil and compared with those of coils having the $10 \%, 22.5 \%, 33 \%$ and $50 \%$ winding arrangements.

Table 1. Details of experimental transformer

\begin{tabular}{lcc}
\hline Transformer Parameter & Designed & Measured \\
\hline Primary coil inductance & $70 \mu \mathrm{H}$ & $70 \mu \mathrm{H}$ \\
Primary coil capacitance & $17.5 \mathrm{nF}$ & $17.5 \mathrm{nF}$ \\
Primary capacitor stored energy & $875 \mathrm{~mJ}$ & $875 \mathrm{~mJ}$ \\
Primary resonant frequency & $145 \mathrm{kHz}$ & $144 \mathrm{kHz}$ \\
Secondary coil inductance & $45 \mathrm{mH}$ & $45 \mathrm{mH}$ \\
Secondary coil capacitance & $10 \mathrm{pF}$ & $9.4 \mathrm{pF}$ \\
Top load capacitance & $21 \mathrm{pF}$ & $20 \mathrm{pF}$ \\
\hline
\end{tabular}

\section{Experimental Results}

Figure 3 compares the spectrum of the reference coil with that of the $10 \%$ coil in terms of the currents flowing in the equivalent circuit of the coil, and the different mode frequencies can be identified. The resonant frequencies and end loaded Q factors of the various mode responses for the experimental coils are recorded in Figure 4.

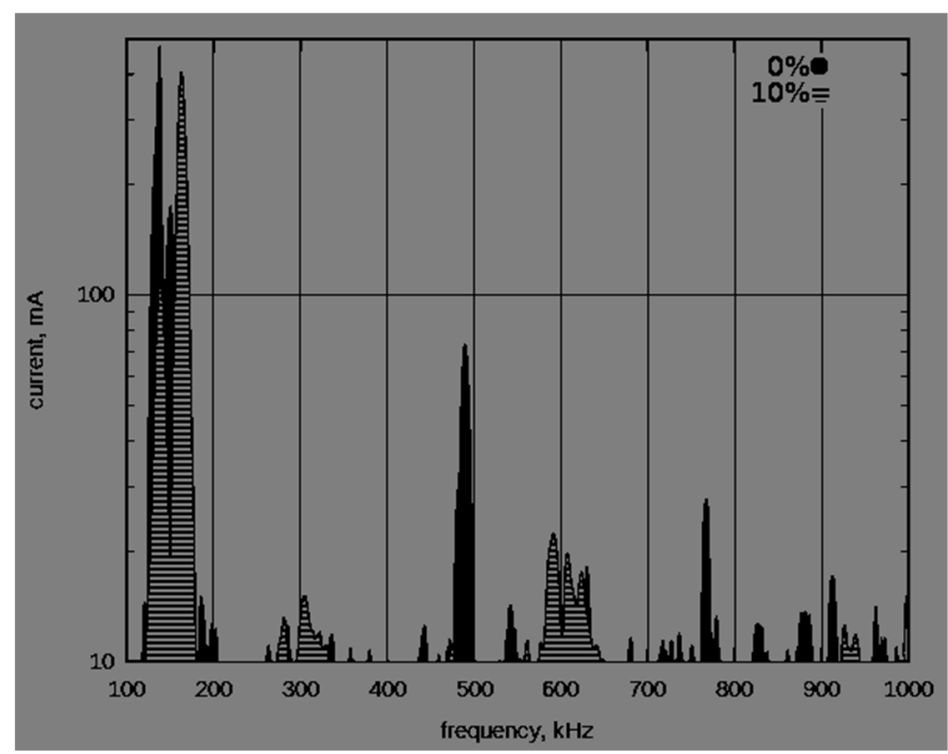

Figure 3 Resonant mode frequencies and $\mathrm{Q}$ factors: $\mathrm{f}_{1}$ modes around $250 \mathrm{kHz}, \mathrm{f}_{3}$ modes around $600 \mathrm{kHz}, \mathrm{f}_{5}$ modes above $800 \mathrm{kHz}$.

Examination of the $\mathrm{Q}$ factor data reveals some interesting details, with the figures showing that the fundamental resonant frequencies and Q factors for all five coils are respectively grouped around $250 \mathrm{kHz}$ and 200 . However, whilst the $\mathrm{f}_{3}$ modes for all the coils show relatively small differences between their frequencies, larger differences in the $Q$ factors are evident. In particular, for the $f_{3}$ response, a significant mean decrease in the $Q$ factor of approximately $11.2 \%$ from the reference coil value was achieved.

When the $10 \%$ and $22.5 \%$ coils are compared, it will be seen that a decrease in the $Q$ factor for both the $f_{3}$ and the $\mathrm{f}_{5}$ modes is observed, whilst for the fundamental frequency there is a minor increase in the Q factors.

Their mean responses are however not significantly different from that of the reference coil. In general, the higher-order modes of the modified coils experience a larger change in their resonant frequencies, whilst the Q factors diminish, both compared with the reference coil. Although for the $10 \%$ coil the higher-mode frequency 
changes are greatest in comparison with those for the reference coil, the associated Q factors are lowest, with the $10 \%$ coil having a reduction of $10 \%$ and the $22.5 \%$ coil a reduction of $8.7 \%$. The single greatest $\mathrm{Q}$ factor reduction is $14 \%$ for the $22.5 \%$ coil for its $\mathrm{f}_{3}$ mode.

During a theoretical investigation into the effects of introducing a reverse-winding section into a solenoid, Vitzmuller (1987) advanced a reason why the fundamental mode frequency remains largely unaffected. Although reverse winding of a section of a coil reduces the effective length it also introduces additional capacitance, thereby lowering the resonant frequency, but with the electrical length of the coil remaining at a quarter of the wavelength of the fundamental mode frequency. Alternatively, when an RF current flows in a coil the effective electrical dimensions become a function of frequency and, for example, the third harmonic becomes $\left(3 \mathrm{f}_{1}+\Delta \mathrm{f}\right)$. A detailed analysis of these modes is not trivial, but it seems likely that the reverse winding process produces interference effects only at certain higher-order modes and not at the fundamental mode (Craven, 2014).

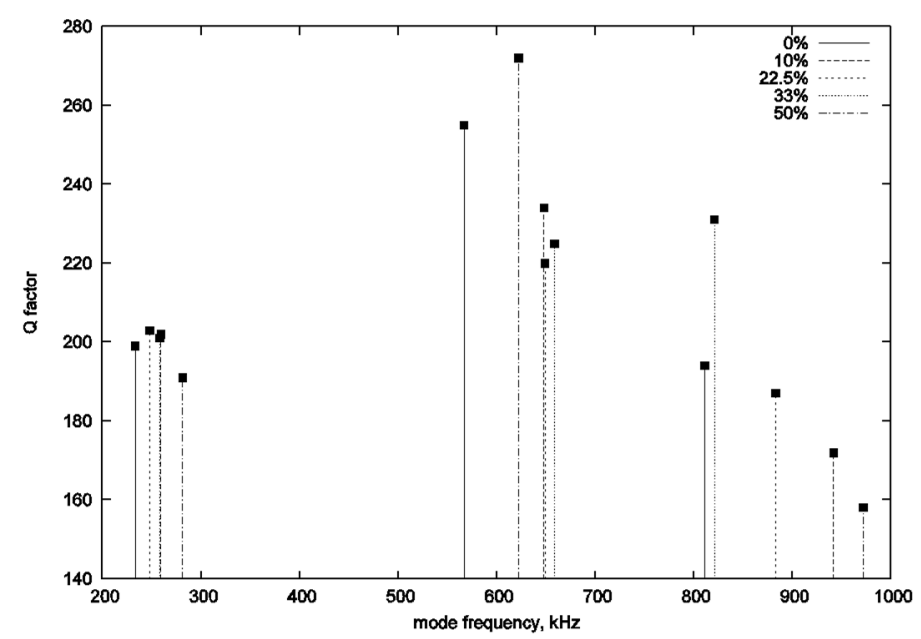

Figure 4. Comparison of the frequency spectra of the reference coil with that of the $10 \%$ coil. Both coils top loaded with a field grading electrode

\section{Conclusion}

Design techniques used in helical cavity filters have been explored and applied to Tesla transformer secondary coils (i.e. single-layer solenoidal resonators). A measurement technique has been described which allows the fundamental and higher mode frequencies and amplitudes of a range of experimental Tesla transformer secondary windings to be characterized. In particular, the authors have demonstrated that the magnitude of currents which flow in the secondary winding at higher mode frequencies can be reduced whilst leaving the fundamental current amplitudes largely unchanged. The combined results achieved in reducing the higher-order currents in the secondary winding and the $\mathrm{Q}$ factor of the winding are sufficiently significant to find utility in such high power applications as portable EMP generators (Sarkar, 2006) or as a pulsed power supply for driving experimental lasers or radio frequency sources, e.g. magnetrons for electronic warfare applications.

\section{References}

Craven, R. M. (2014). A study of secondary windings for the two-coil Tesla transformer (Doctoral thesis) Loughborough University, Leicestershire, UK.

Efremov, A. M., Koshalev, V. I., Kovalchuk, B. M., Plisko, V. V., \& Sukhushin, K. N. (2011). High-power sources of ultra-violet radiation with subnanosecond pulse length, Instrum. Experimen. Tech., 54(1), 70-76.

Glascoe, G. N., \& Lebacqz, J. V. (1948). Pulse Generators. McGraw-Hill, USA.

Sargeant, W. J., \& Dollinger, R. E. (1989). High Power Electronics. New York: TAB Professional and Reference Books.

Sarkar, P., Braidwood, S. W., Smith, I. R., Novac, B. M., Miller, R. A., \& Craven, R. M. (2006). A compact battery-powered half-megavolt transformer system for EMP generation. IEEE Transactions on Plasma Science, 34(5), 1832-1837. 
Terman, F. Radio Engineer's Handbook, McGraw-Hill, USA.

Vitzmuller, P. (1987). Filters with Helical and Folded Resonators. Norwood, MA, USA, Artech House.

Wang, M., Novac, B. M., Pecastaing, L., \& Smith, I. R. (2016). Bipolar Modulation of the Output of a 10-GW Pulsed Power Generator. IEEE Transactions on Plasma Science, 44(6), 1971-1977.

Zhao, L., Pan, Y. F., Zhang, X. B.,Wang, L. M., Fang, J. P., Sun, X., \& Lui, R. (2013). A Tesla-type repetitive pulse generator for solid dielectric research. Review of Scientific Instruments, 84(10), 105-114

Zverev, A. I. (1967). Handbook of Filter Synthesis. New York, NY, USA, Wiley.

\section{Copyrights}

Copyright for this article is retained by the author(s), with first publication rights granted to the journal.

This is an open-access article distributed under the terms and conditions of the Creative Commons Attribution license (http://creativecommons.org/licenses/by/4.0/). 